\title{
Mean Platelet Volume is Associated with Coronary Artery Disease and Cardiovascular Risk Factors, but Platelet Distribution Width Not
}

\author{
Sinem N1*, Ahmet Goktug E2 ${ }^{2}$ Engin $\mathrm{S}^{3}$ and Tolga Han E $\mathrm{E}^{4}$ \\ ${ }^{1}$ Department of Haematology, Dr Abdurrahman Yurtaslan Ankara Oncology Education \\ and Research Hospital, Turkey \\ ${ }^{2}$ Department of Cardiology, Ankara Penal Institution Campus State Hospital, Turkey \\ ${ }^{3}$ Department of Internal Medicine, Ankara Numune Education and Research Hospital, \\ Turkey
}

${ }^{4}$ Department of Cardiology, Mus State Hospital, Turkey

*Corresponding author: Sinem Namdaroglu, Department of Haematology, Dr. Abdurrahman Yurtaslan Ankara Oncology Education and Research Hospital, 06200, Ankara, Murat Mah Gezegen Sok No: 11/6 Cankaya/ Ankara/ Turkey, Postal Code: 06290, Turkey, Tel: 00905332266616; Email: drsinemnamdaroglu@gmail.com

\section{Abstract}

Objective: Coronary atherosclerosis, the primary cause of coronary artery disease, is a multifactorial disease, the etiology of which involves interaction of many humoral and cellular factors. It has been also known that platelets play a pivotal role in atherothrombosis. In this study, we aimed to find out whether there is a correlation between mean platelet volume levels (MPV), platelet distribution width (PDW) levels and coronary artery disease (CAD), other diseases that may cause cardiovascular risks and to determine the relationship between high-sensitivity C-reactive protein (hs-CRP), MPV and PDW.

Materials and Methods: We included 132 patients with CAD, who were diagnosed by angiography, and 82 control subjects. We evaluated MPV, PDW, and hs-CRP levels, and determined the association between these levels with cardiovascular risk factors.

Results: MPV levels of the patients group were found significantly lower than the control group levels. $(p=0.041)$. There were no significant difference between the patients group and control group in terms of PDW values ( $\mathrm{p}=0.332)$. The hsCRP levels of the patients group was found significantly higher than the control group levels $(\mathrm{p}=0.010)$. In patients group there were found no significant correlation between hs-CRP levels and platelet indices levels.

Conclusion: In conclusion, MPV was lower in CAD patients compared with control subjects, but PDW had no significant. There were association between MPV, and number of chronic disease. After correlation analysis, we found negative and 


\section{Medical Journal of Clinical Trials \& Case Studies}

weak correlation between platelet levels and MPV levels in CAD group. These findings may be related to our small sample size. There is a need for large-scale and new studies, to gain powerful data.

Keywords: Coronary artery disease; Platelet indices; High sensitive C-reactive protein

Abbreviation: MPV: Mean Platelet Volume Levels; PDW: Platelet Distribution Width; CAD: Coronary Artery Disease; ASVD: Atherosclerotic vascular diseases; MI: myocardial infarction; hsCRP: High-Sensitivity C-Reactive Protein; HT: Hypertension; DM: Diabetes Mellitus; HL: Hyperlipidemia; CBC: Complete Blood Count; TC: total cholesterol; HDL-C: HDL cholesterol; TG: triglyceride; LDL-C: LDL cholesterol; BUN: Blood Urea Nitrogen; RDW: Red Cell Distribution Width; PTCA: Percutaneous Transluminal Coronary Angiography; CRP: C Reactive Protein.

\section{Introduction}

Atherosclerosis is the most important pathological result of the mechanisms that is underlying coronary artery disease (CAD). It is an inflammatory fibroproliferative response mediated against various forms of endothelial damage. Atherosclerotic vascular diseases (ASVD) is the major cause of morbidity and mortality $[1,2]$.

Antiplatelet drugs reduce the risk of cardiovascular events; therefore platelets have one the major role in the atherothrombosis process [3]. It is suggested that platelet activity has an important role in myocardial infarction (MI), which was an outcome of prothrombotic process, and the measurement of platelet activity and/or aggregation can provide prognostic information for the risk of cardiovascular events [4-7]. With understanding of atherosclerosis is an inflammatory process [129] highsensitivity C-reactive protein (hsCRP), as well as many inflammatory plasma markers are defined as potential tools for the interpretation of the cardiovascular disease (CVD) risk $[8,9]$.

The aim of this study was to find out whether mean platelet volume levels (MPV), platelet distribution width (PDW) and hsCRP levels are higher than those seen at the control examinations of patients with CAD and to determine whether chronic diseases (eg, diabetes mellitus (DM), hypertension (HT), hyperlipidemia (HL), and obesity, etc.) that are associated with cardiovascular risk factors have an effect on MPV, PDW, and on hsCRP levels.

\section{Materials and Methods}

\section{Study Design}

This is a retrospective observational study. Patients who were admitted to cardiology clinic between March 2009 and May 2009 and at whom CAD was diagnosed were included into the study. For the patients group of the study, patients at whom CAD was diagnosed by coronary angiography according to hospital records $(n=132)$ and for control group of the study, patients at whom no findings found $(n=82)$ were taken into our study randomly. Complete blood count (CBC) and platelet indices were measured by auto analyzer (Beckman Coulter LH780 Hematology Analyzer with LH and LH Slide Maker Slide Stainer). After at least 8 hours of fasting, biochemical parameters (fasting blood glucose, total cholesterol (TC), HDL cholesterol (HDL-C), triglyceride (TG) levels), hs-CRP were measured (Olympus AU 600 auto analyzer, the latter with Beckman Coulter, Fullerton CA, USA). LDL cholesterol (LDL-C), was calculated with the Friedewald formula [TC-LDL (VLDL + HDL) VLDL $=$ TG /5] by using these values [10].

Patients who had blood disease that may cause any change in platelet functions and distributions, platelet function disorders and active infection that may lead to significant change in hs-CRP levels, malignancy, connective tissue diseases, immunological, and rheumatologic disease were excluded. Selective coronary angiography was performed with femoral approach with Judkins catheters (Philips, 30 square/second, on $35 \mathrm{~mm}$ cine film, 6-7 F guide catheters). For each vessel and left main coronary artery, stenosis of $\% 50$ or above was considered significant. Chronic diseases, such as diabetes mellitus (DM), hypertension (HT), hyperlipidemia (HL), and obesity which may cause cardiovascular risks were evaluated in our study. All of the participants gave informed consent and the local research ethics committee had previously approved the study protocol.

\section{Statistical Analysis}

All the data obtained from the study were analyzed using SPSS 16.0 on computer. The results obtained in 


\section{Medical Journal of Clinical Trials \& Case Studies}

continuous variables were expressed in forms of the average (mean) \pm standard deviation (SD). After descriptive statistics were analyzed (frequency, percentage distribution, mean \pm standard deviation), compliance with the normal distribution of variables assessed by Kolmogorov-Smirnov One Sample Test. For biochemical parameters of blood count levels of two groups, Student's $t$ test was used in the data meeting the assumptions of parametric variables and Mann-Whitney $\mathrm{U}$ test was used in those which were nonparametric. Kruskal Wallis test was used for comparing three groups and Mann-Whitney $U$ test was used for comparing two groups where necessary. For $\mathrm{P}$ values where $\mathrm{P}<0.05$ was considered as statistically significant.

\section{Results}

Demographic and biochemical characteristics of the patients with CAD and the controls are shown in Table 1. White blood cell, serum creatinin levels, blood urea nitrogen (BUN) levels, and serum fasting glucose levels were significantly higher in CAD patients. In addition, HDL cholesterol levels were significantly lower in CAD patients. There were no significant differences in hemoglobine levels, red cell distribution width (RDW), and platelet counts between the groups $(p=0.126$, $\mathrm{p}=0.332, \mathrm{p}=0.205$, respectively). The MPV was significantly lower in the CAD compared with control group $(p=0.041)$. The hs-CRP was found significantly higher the control group $(p=0,010)$. There were found no statistically significant difference between two groups in terms of PDW $(\mathrm{p}=0.332)$, and INR (international normalized ratio) ( $\mathrm{p}=0.332, \mathrm{p}=0.233$, respectively).

In CAD group, there were found statistically significant differences in MPV values between the group without chronic disease, the group with one chronic disease, and the group with 2 or more than 2 chronic diseases $(p=0.004)$. The MPV values of the group without chronic disease, and the group with one chronic disease were lower than the MPV values of the group with $\geq 2$ chronic diseases $(p=0.030, p=0.002$, respectively). There were found no significant difference between three groups in terms of PDW, hs-CRP, and INR levels ( $\mathrm{p}=0.509, \mathrm{p}=0.581$, $\mathrm{p}=0.262$, respectively) (Table 2 ).

After correlation analysis, as seen in Table 3, we found negative and weak correlation between platelet levels and MPV levels in CAD group $(\mathrm{r}=-0.429, \mathrm{p}<0.001)$, there were found positive weak correlation between HDL-C values, and MPV values ( $\mathrm{r}=0.172, \mathrm{p}=0.048)$, and there were found negative weak correlation between HDL-C, and PDW values.

\begin{tabular}{|c|c|c|c|c|c|}
\hline & \multirow{2}{*}{\multicolumn{2}{|c|}{$\begin{array}{l}\text { Control } \\
(n=82)\end{array}$}} & \multirow{2}{*}{\multicolumn{2}{|c|}{ Coronary artery disease group $(n=132)$}} & \multirow{3}{*}{ p value } \\
\hline & & & & & \\
\hline & mean & SD & Mean & SD & \\
\hline Age ( year) & 58.01 & 10.32 & 60.36 & 10.75 & $0,116^{* *}$ \\
\hline Gender (male, \%) & $33(40.2 \%)$ & & $71(53.8 \%)$ & & $0,074 * * *$ \\
\hline Smoking (\%) & $24(29.6 \%)$ & & $40(30.8 \%)$ & & $1.00^{* * *}$ \\
\hline $\mathrm{WBC}\left(x 10^{3} / \mathrm{ml}\right)$ & 7.66 & 2.71 & 8.36 & 2.89 & $0.022^{*}$ \\
\hline Hemoglobine $(g / d L)$ & 13.62 & 1.78 & 14 & 1.77 & $0.126^{* *}$ \\
\hline RDW & 14.15 & 1.82 & 14.28 & 2.09 & $0.332^{* *}$ \\
\hline Platelet $\left(x 10^{3} / \mathrm{ml}\right)$ & 270.68 & 61.46 & 259.21 & 65.84 & $0.205^{* *}$ \\
\hline BUN (mg/dl) & 29.01 & 11.35 & 32.91 & 13.85 & $0.026^{*}$ \\
\hline Creatinine $(\mathrm{mg} / \mathrm{dl})$ & 0.73 & 0.18 & 0.89 & 0.36 & $<0.001 *$ \\
\hline AST (U/L) & 23.26 & 7.72 & 27.55 & 28.3 & $0.586^{*}$ \\
\hline ALT (U/L) & 22.43 & 10.02 & 22.58 & 14.51 & $0.423^{*}$ \\
\hline Glucose (mg/dl) & 97.05 & 31.53 & 106.75 & 39.85 & $0.042 *$ \\
\hline Total chol. (mg/dl) & 180.79 & 34.04 & 185.3 & 39.04 & $0.391^{* *}$ \\
\hline LDL chol. (mg/dl) & 115.37 & 29.28 & 122.08 & 34.85 & $0.149^{* *}$ \\
\hline HDL chol. (mg/dl) & 40.33 & 13.78 & 37.93 & 25.81 & $0.004^{*}$ \\
\hline Triglyseride $(\mathrm{mg} / \mathrm{dl})$ & 147.56 & 100.88 & 149.19 & 67.73 & $0.154^{*}$ \\
\hline MPV(fl) & 8.64 & 0.98 & 8.37 & 0.93 & $0.041^{* *}$ \\
\hline PDW & 16.34 & 0.65 & 16.43 & 0.72 & $0.332^{*}$ \\
\hline
\end{tabular}




\section{Medical Journal of Clinical Trials \& Case Studies}

\begin{tabular}{|c|c|c|c|c|c|}
\hline hsCRP(mg/dl) & $\mathbf{1 . 4 3}$ & $\mathbf{2 . 6 7}$ & $\mathbf{3 . 4 9}$ & $\mathbf{1 0 . 2 7}$ & $\mathbf{0 . 0 1 0}^{*}$ \\
\hline INR & 1.01 & 0.1 & 1.04 & 0.18 & $0.233^{*}$ \\
\hline
\end{tabular}

WBC: white blood cell, RDW: red cell distribution, Chol: cholesterole, MPV: mean platelet volume, PDW: platelet distribution width, hsCRP: high sensitive CRP.

SD: Standard deviation

* Mann-Whitney U Test

** Student t Test

*** chi square test

Table 1: Clinical and demographical characteristics of the patients and the study subjects.

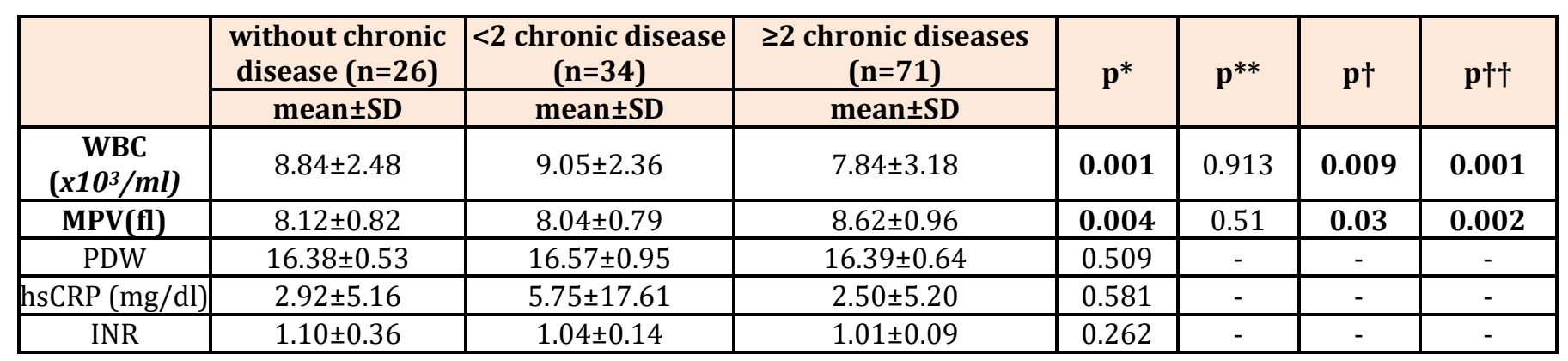

WBC: white blood cell, MPV: mean platelet volume, PDW: platelet distribution width, hsCRP: high sensitive CRP.

SD: Standard deviation

*Kruskal-Wallis test

**Comparison of the patients without chronic disease and the patients with $<2$ chronic disease (Mann Whitney U Testi) †Comparison of the patients without chronic disease and the patients with $\geq 2$ chronic diseases (Mann Whitney U Testi) ††Comparison of the patients with $<2$ chronic disease and the patients with $\geq 2$ chronic disease (Mann Whitney U Testi) Table 2: Comparison of white blood cell, platelet indices, and hsCRP levels of the groups without chronic disease, with < 2 chronic diseases, and with $\geq 2$ chronic diseases.

\begin{tabular}{|c|c|c|c|c|}
\hline & & MPV & PDW & hs-CRP \\
\hline \multirow{2}{*}{ Platelet } & $\mathbf{r}$ & $\mathbf{- 0 . 4 2 9}$ & & \\
\cline { 2 - 5 } & $\mathbf{p}$ & $<\mathbf{0 . 0 0 1}$ & & \\
\hline \multirow{2}{*}{ PDW } & $\mathrm{r}$ & -0.003 & & \\
\cline { 2 - 5 } & $\mathrm{p}$ & 0.971 & -0.014 & \\
\hline \multirow{2}{*}{ hsCRP } & $\mathrm{r}$ & -0.115 & 0.888 & -0.136 \\
\hline \multirow{2}{*}{ glucose } & $\mathrm{p}$ & 0.255 & 0.105 & 0.176 \\
\hline \multirow{2}{*}{ Total chol. } & $\mathrm{r}$ & 0.034 & 0.232 & -0.064 \\
\hline \multirow{2}{*}{ LDL chol. } & $\mathrm{p}$ & 0.695 & -0.053 & -0.528 \\
\hline \multirow{2}{*}{ HDL chol. } & $\mathrm{r}$ & 0.08 & 0.547 & 0.645 \\
\hline \multirow{2}{*}{ Trygliseride } & $\mathrm{p}$ & 0.363 & -0.045 & -0.105 \\
\cline { 2 - 5 } & $\mathrm{p}$ & 0.085 & $\mathbf{- 0 . 1 9 1}$ & 0.299 \\
\hline & $\mathbf{r}$ & 0.332 & $\mathbf{0 . 0 2 8}$ & -0.087 \\
\hline
\end{tabular}

PDW: platelet distribution width, chol.: cholesterole, hsCRP: high sensitive CRP.

*Spearman's correlation test.

Table 3: Correlations of platelet indices, hsCRP, and biochemical parameters in the study group. 


\section{Medical Journal of Clinical Trials \& Case Studies}

\section{Discussion}

Platelet activation and aggregation measurements can provide prognostic information for the risk cardiovascular events [6,7]. Large volume platelets are more active in terms of metabolic and enzymatic [11]. In addition, the sizes of platelets are consistent with their prothrombotic potentials [12]. Although there has not been any specific measurement method (international standardization) for MPV that is an indicator of platelet sizes, it is routinely used as a cheap assessment tool that implemented both hospitalized patients and outpatients. It is considered that the ex vivo measurements of platelet functions are a reflection of platelet's functional capacity, and it is shown that increase in MPV is associated with platelet aggregation, increased thromboxane synthesis, release of $\beta$-thromboglobulin, and platelet markers such as increased expression of adhesion molecules $[5,13]$.

The increased MPV levels are debatable as a risk factor for coronary artery disease (CAD) [14]. Endler, et al. showed that patients who were diagnosed CAD and whose MPV levels were ( $>11.6 \mathrm{fl})$, have a high risk in terms of MI [15]. In patients at whom percutaneous transluminal coronary angiography (PTCA) was done in emergency conditions, it was found that the number of platelets was low while the MPV levels were higher [16]. According to the two different studies' results, it is reported that in patients with acute MI and unstable angina pectoris, MPV and PDW were higher than the controls [17]. In one study, MPV and PDW levels were higher in the patients with no coronary lesions than the patiens with coronary lesion [18]. We found that MPV value of the $\mathrm{CAD}$ patients was lower than the control group. There was no significant difference between two groups in terms of PDW values.

C reactive protein (CRP) has two distinct conformations: pentameric (p) CRP and monomeric (m) CRP [19]. It has been shown that pCRP get increased in chronic conditions such as ASVD, HT, metabolic syndrome and type 2 DM [20]. The transformation of pCRP to mCRP is done by activated platelets and as a result mCRP accumulates in atherosclerotic plaque [19]. CRP is a strong predictor for cardiovascular events that will occur in the future. The relationship between high CRP levels and arterial disease was first shown in 1997 by Ridker, et al. [21]. Ridker, et al. found that CRP is a strong predictor of myocardial infarction and stroke in healthy asymptomatic men. The results of large number of studies done subsequently, support the conclusions found in this study [22-24]. In our study, hs-CRP values of the CAD group were found significantly higher than the control group. Although we didn't measure the molecular fractions (mCRP ve pCRP) of CRP in our study, the finding of hs-CRP levels in CAD group being higher than control group supports the previous evidences that this inflammatory marker has a role in the process of atherosclerosis.

Although it has been reported that MPV has no correlation with known ischemic heart disease risk factors, DM, HT, HL, it is found higher when circumstances that caused cardiovascular risks such as smoking, and obesity are seen [25-30]. The results of our study has shown that MPV values of the group without chronic diseases and group with one chronic disease are lower than the MPV values of the group with 2 or more than two chronic disease. Furthermore, we observed that chronic diseases singly (HT or HL) did not cause a significant increase in MPV levels. In this context, as the number chronic diseases increase, inflammatory processes underlying the increased cardiovascular risk becomes stronger, and it may affect the platelet activity in favor of atherosclerosis in a negative way, however, there were no significant difference among these three groups in terms of PDW values.

\section{Study Limitations}

One of the limitations of this study is having been reached the data as retrospective. The second one is small sample size.

\section{Conclusion}

MPV levels in CAD group were significantly lower than the control group levels. There were seen no significant differences between PDW, and INR values of the two groups. We found a weak negative correlation between number of platelets in CAD group and MPW levels. In order to obtain more robust data new large-scale studies are needed in order to obtain data.

\section{References}

1. Peterson S, Peto V, Rayner M (2005) European Cardiovascular Disease Statistics. London: British Heart Foundation.

2. American Heart Association (2003) Heart disease and stroke statistics. Dallas, TX: American Heart Association.
Sinem N, et al. Mean Platelet Volume is Associated with Coronary Artery Disease and Cardiovascular Risk Factors, but Platelet Distribution Width Not. Med J Clin Trials Case Stud 2018, 2(4): 000174.
Copyright(C) Sinem N, et al. 


\section{Medical Journal of Clinical Trials \& Case Studies}

3. Meadows TA, Bhatt DL (2007) Clinical aspects of platelet inhibitors and thrombus formation. Circ Res 100(9): 1261-1275.

4. Haver VM, Gear AR (1981) Functional fractionation of platelets. J Lab Clin Med 97(2): 187-204.

5. Bath PM, Butterworth RJ (1996) Platelet size: measurement, physiology and vascular disease. Blood Coagul Fibrinolysis 7(2): 157-161.

6. Aul KA, Cannon CP, Mitchell J, McCahan J, Tracy RP, et al. (1999) Platelet activation in patients after an acute coronary syndrome: results from the TIMI-12 trial. Thrombolysis in Myocardial Infarction. J Am Coll Cardiol 33(3): 634-639.

7. Trip MD, Cats VM, van Capelle FJ, Vreeken J (1990) Platelet hyperreactivity and prognosis in survivors of myocardial infarction. N Engl J Med 322(22): 15491554.

8. Pasceri V, Willerson JT, Yeh ET (2000) Direct proinflammatory effect of C-reactive protein on human endothelial cells. Circulation 102(18): 21652168.

9. Kuller LH, Tracy RP, Shaten J, Meilahn EN (1996) Relation of C-reactive protein and coronary heart disease in the MRFIT nested case-control study. Multiple Risk Factor Intervention Trial. Am J Epidemiol 144(6): 537-547.

10. Nauc M, Warnick GR, Rifai N (2002) Methods for measurement of LDL-cholesterol: a critical assessment of direct measurement by homogeneous assays versus calculation. Clin Chem 48(2): 236-254.

11. Karpatkin S (1969) Heterogeneity of human platelets. II. Functional evidence suggestive of young and old platelets. J Clin Invest 48(6): 1083-1087.

12. Kamath S, Blann AD, Lip GY (2001) Platelet activation: assessment and quantification. Eur Heart J 22(17): 1561-1571.

13. Gurbel PA, Becker RC, Mann KG, Steinhubl SR, Michelson AD (2007) Platelet function monitoring in patients with coronary artery disease. J Am Coll Cardiol 50(19): 1822-1834.

14. Halbmayer WM, Haushofer A, Radek J, Schön R, Deutsch M, et al. (1995) Platelet size, fibrinogen and lipoprotein(a) in coronary heart disease. Coron Artery Dis 6(5): 397-402.

15. Endler G, Klimesch A, Sunder-Plassmann H, Schillinger M, Exner M, et al. (2002) Mean platelet volume is an independent risk factor for myocardial infarction but not for coronary artery disease. Br J Haematol 117(2): 399-404.

16. Butkiewicz AM, Kemona H, Dymicka-Piekarska V, Bychowski J (2003) Beta-thromboglobulin and platelets in unstable angina. Kardiol Pol 58(6): 449455.

17. Khandekar MM, Khurana AS, Deshmukh SD, Kakrani AL, Katdare AD, et al. (2006) Platelet volume indices in patients with coronary artery disease and acute myocardial infarction: an Indian scenario. J Clin Pathol 59(2): 146-149.

18. Ihara A, Kawamoto $T$, Matsumoto $K$, Shouno $S$, Hirahara C, et al. (2006) Relationship between platelet indexes and coronary angiographic findings in patients with ischemic heart disease. Pathophysiol Haemost Thromb 35(5): 376-379.

19. Eisenhardt SU, Habersberger J, Murphy A, Chen YC, Woollard KJ, et al. (2009) Dissociation of pentameric to monomeric C-reactive protein on activated platelets localizes inflammation to atherosclerotic plaques. Circ Res 105(2): 128-137.

20. Jialal I, Devaraj S, Venugopal SK (2004) C-reactive protein: risk marker or mediator in atherothrombosis?. Hypertension 44(1): 6-11.

21. Ridker PM, Cushman M, Stampfer MJ, Tracy RP, Hennekens CH (1997) Inflammation, aspirin, and the risk of cardiovascular disease in apparently healthy men. N Engl J Med 336(14): 973-979.

22. Koenig W, Löwel H, Baumert J, Meisinger C (2004) Creactive protein modulates risk prediction based on the Framingham Score: implications for future risk assessment: results from a large cohort study in southern Germany. Circulation 109(11): 1349-1353.

23. Pai JK, Pischon T, Ma J, Manson JE, Hankinson SE, et al. (2004) Inflammatory markers and the risk of coronary heart disease in men and women. $\mathrm{N}$ Engl J Med 351(25): 2599-2610. 


\section{Medical Journal of Clinical Trials \& Case Studies}

24. Best LG, Zhang Y, Lee ET, Yeh JL, Cowan L, et al. (2005) C-reactive protein as a predictor of cardiovascular risk in a population with a high prevalence of diabetes: the Strong Heart Study. Circulation 112(9): 1289-1295.

25. Huczek Z, Kochman J, Filipiak KJ, Horszczaruk GJ, Grabowski M, et al. (2005) Mean platelet volume on admission predicts impaired reperfusion and longterm mortality in acute myocardial infarction treated with primary percutaneous coronary intervention. J Am Coll Cardiol 46(2): 284-290.

26. Papanas N, Symeonidis G, Maltezos E, Mavridis G, Karavageli E, et al. (2004) Mean platelet volume in patients with type 2 diabetes mellitus. Platelets 15(8): 475-478.
27. Nadar S, Blann AD, Lip GY (2004) Platelet morphology and plasma indices of platelet activation in essential hypertension: effects of amlodipine-based antihypertensive therapy. Ann Med 36(7): 552-557.

28. Pathansali R, Smith N, Bath P (2001) Altered megakaryocyte-platelet haemostatic axis in hypercholesterolaemia. Platelets 12(5): 292-297.

29. Kario K, Matsuo T, Nakao K (1992) Cigarette smoking increases the mean platelet volume in elderly patients with risk factors for atherosclerosis. Clin Lab Haematol 14(4): 281-287.

30. Coban E, Ozdogan M, Yazicioglu G, Akcit F (2005) The mean platelet volume in patients with obesity. Int J Clin Pract 59(8): 981-982. 